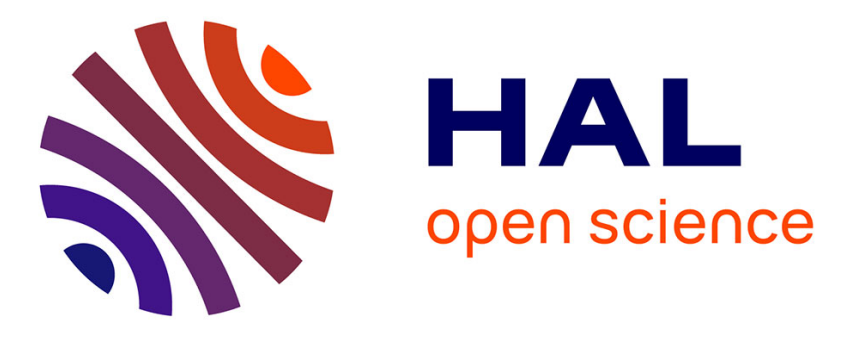

\title{
A $7 \mathrm{Mb}$ region within 11q13 may contain a high penetrance gene for breast cancer
}

Juan Manuel Rosa-Rosa, Guillermo Pita, Anna González-Neira, Roger L.

Milne, Victoria Fernandez, Claudia Ruivenkamp, Christi J. Asperen, Peter

Devilee, Javier Benitez

\section{To cite this version:}

Juan Manuel Rosa-Rosa, Guillermo Pita, Anna González-Neira, Roger L. Milne, Victoria Fernandez, et al.. A $7 \mathrm{Mb}$ region within 11q13 may contain a high penetrance gene for breast cancer. Breast Cancer Research and Treatment, 2009, 118 (1), pp.151-159. 10.1007/s10549-009-0317-1 . hal-00535336

\section{HAL Id: hal-00535336 https://hal.science/hal-00535336}

Submitted on 11 Nov 2010

HAL is a multi-disciplinary open access archive for the deposit and dissemination of scientific research documents, whether they are published or not. The documents may come from teaching and research institutions in France or abroad, or from public or private research centers.
L'archive ouverte pluridisciplinaire HAL, est destinée au dépôt et à la diffusion de documents scientifiques de niveau recherche, publiés ou non, émanant des établissements d'enseignement et de recherche français ou étrangers, des laboratoires publics ou privés. 


\title{
A $7 \mathrm{Mb}$ region within $11 \mathrm{q} 13$ may contain a high penetrance gene for breast cancer
}

\author{
Juan Manuel Rosa-Rosa - Guillermo Pita • Anna González-Neira • \\ Roger L. Milne - Victoria Fernandez · Claudia Ruivenkamp • \\ Christi J. van Asperen · Peter Devilee · Javier Benitez
}

Received: 15 October 2008/ Accepted: 13 January 2009/Published online: 10 February 2009

(C) Springer Science+Business Media, LLC. 2009

\begin{abstract}
Familial breast cancer represents up to 5\% of all breast cancer cases. Recently, our group has performed a new SNP-based linkage study in 19 non-BRCA1/2 families. We found that a single family was linked to regions in two different chromosomes (11q13 and 14q21), and observed a non-parametric LOD score of 11.5 in both regions. In the present study, we ruled out any possible translocation between the chromosomes. We also used both a panel of STRs and an indirect approach based on HapMap data to narrow down these regions from 28 to $7 \mathrm{Mb}$ in
\end{abstract}

Electronic supplementary material The online version of this article (doi:10.1007/s10549-009-0317-1) contains supplementary material, which is available to authorized users.

J. M. Rosa-Rosa · V. Fernandez $\cdot$ J. Benitez $(\bowtie)$

Human Genetics Group, Human Cancer Genetics Programme,

Spanish National Cancer Research Centre (CNIO), Melchor

Fernandez Almagro 3, Madrid 28029, Spain

e-mail: jbenitez@cnio.es

G. Pita · A. González-Neira · R. L. Milne · J. Benitez

Genotyping Unit (CeGen), Human Cancer Genetics Programme,

Spanish National Cancer Research Centre, Madrid, Spain

C. Ruivenkamp

Department of Diagnostic Genome Analysis, Leiden University

Medical Center, Leiden, The Netherlands

C. J. van Asperen

Department of Clinical Genetics, Leiden University Medical

Center, Leiden, The Netherlands

P. Devilee

Department of Human Genetics, Leiden University Medical

Center, Leiden, The Netherlands

J. Benitez

Centro de Investigaciones de Enfermedades Raras (CIBERER),

Valencia, Spain chromosome 11 and from 14.5 to $8.5 \mathrm{Mb}$ in chromosome 14. We performed a mutational screening on candidate genes in 11q13 (NUMA1, FGF3, CCND1, RAD9A, RNF121, FADD and hsa-mir-192), and on FOXAl in $14 \mathrm{q} 21$. Although we have not found any deleterious mutations in the coding region of these genes, data from STR markers confirm 11q13 as a candidate region to contain a breast cancer susceptibility gene.

Keywords Breast cancer - Linkage study - Haplotype · Singlepoint LOD score

\section{Introduction}

Familial breast cancer represents up to 5\% of all breast cancer cases [1]. The two known high-penetrance genes for breast cancer, BRCA1 and BRCA2, are involved in about $25 \%$ of these families [2-5]. The remaining families do not carry mutations in these genes and are known as BRCAX families.

While several candidate regions to carry a susceptibility gene for breast cancer have been identified by linkage studies based on microsatellite (STR) [6-8] or SNP markers [9], no high-penetrance gene has been found since $B R C A 1$ and BRCA2 were cloned.

There are a number of possible explanations for the lack of consistency in these results. One is the high heterogeneity within BRCAX cases suggested by some authors [10] and described by immunohistochemical studies [11]. Another is that susceptibility loci might be populationspecific. Recently, our group performed a new SNP-based linkage study in 19 BRCAX families from Spain, The Netherlands and USA. In addition to demonstrating that SNPs have more power than STRs for linkage detection 
[12], five candidate regions were described on chromosomes 2, 4, 7, 11 and 14, consistent with the existence of high heterogeneity within BRCAX families. A single family (FAM153) was linked to two of these regions (11q12.3q14.3 and 14q13.1q21.3), with a multipoint parametric LOD score under a dominant model (multipoint HLOD score) of 2.20, and non-parametric LOD scores of 11.50 (multipoint) and 4.50 (two points), in each region. These high LOD scores may be due to the fact that this family (FAM153) is large and very informative.

In the present study, we have performed a mutational screening of literature-based candidate genes in both these regions in this family. Although we have not found any mutations in the coding region of the genes studied, we have narrowed down the boundaries for both candidate regions and described different polymorphisms in both coding and non-coding regions.

\section{Materials and methods}

Familial cases and controls

The familial structure of FAM153 is shown in Fig. 1. FAM153 is a large family consisting of eighteen members from four generations, with six females affected with breast cancer. The family members available for the genotyping analysis were II-7, IV-1, IV-2 and IV-3.

Index cases from 125 high-risk breast cancer families (BRCAX) recruited through the CNIO were included to evaluate the frequency of rs11278712 (see Gene study section). These high-risk families contained at least twofirst-degree relative females affected with breast cancer (at least one of them diagnosed before 50), or at least three affected women of more distant kinship. DNA extracted from peripheral blood from the 125 index cases had been fully screened for mutations in $B R C A 1$ and $B R C A 2$ by a combination of DHPLC and direct sequencing, without detecting any deleterious mutations.

In addition, DNA samples from 465 non-related Spanish women aged between 40 and 65 and without personal or familial antecedents of any type of cancer were analyzed to establish the frequency of rs11278712 in the general population.

\section{Candidate regions}

The candidate region on chromosome 11 identified in our previous study [12] spans from rs 1525064 to rs1388472, or over $28 \mathrm{Mb}$ (Fig. 1a), while that on chromosome 14 spans from rs2027338 to rs 1532202 , covering around $14.5 \mathrm{Mb}$ (Fig. 1b). Both regions gave a multipoint HLOD score of 2.20 [12].
In order to confirm and narrow down these regions, we performed a fine-mapping study based on STRs. We genotyped a total of 11 STRs (D11S4174, D11S4191, D11S4076, D11S1889, D11S4136, D11S4081, D11S4172, D11S901, D11S4147, D11S1367 and D11S873) covering and flanking the candidate region in chromosome 11 and 6 STRs (D14S1034, D14S70, D14S1014, D14S129, D14S 288 and D14S269) covering and flanking the candidate region in chromosome 14 (Fig. 1). We calculated the singlepoint HLOD score for each marker using Merlin software [13].

\section{Significance testing}

We estimated the empirical probability of obtaining two peaks with multipoint HLOD $>2.20$ in the same family by chance alone, by performing a simulation study based on our set of 19 BRCAX families [12]. This was carried out using Merlin software.

The probability that two independent loci segregate together in a family was calculated assuming that these two loci came from an unknown single ancestor, as:

$p=2 \times(1 / 4)^{n}$, where $n$ is the total number of meioses.

\section{Cytogenetic and FISH analyses}

In order to rule out the possibility that a germinal translocation between chromosomes 11 and 14 segregated in FAM153, two analyses were performed on peripheral blood cells from one member of the family: a conventional GTG-banding technique, and FISH analysis. The FISH analysis was performed using DNA probes specific for the subtelomere of the long arm of chromosome 14 (GS$820 \mathrm{M} 16$, red) and the centromere of chromosome 11 (CEP11, green, Vysis, Inc. Downers Grove, IL, USA), and DNA probes specific for the subtelomere of the long arm of chromosome 11 (GS-26N8, red) and the short arm of chromosome 11 (GS-908H22, green) in order to exclude a cryptic translocation $t(11 ; 14)$. These techniques were performed according to the standard procedures in our laboratories.

\section{HapMap-based haplotype study}

Due to the large size of the haplotypes shared by the four members studied in FAM153 (28 Mb for 11q13 and 14.5 $\mathrm{Mb}$ for $14 \mathrm{q} 21$ ), we sought to narrow down the region using an approach to identify possible recombination spots in the chromosomes in earlier generations. This approach was based on the idea that the haplotype shared by this family is the result of recombination events that occurred between the ancestral chromosome and other chromosomes, 
Fig. 1 Representation of the HLOD score and the common haplotypes shared by FAM153. Shadowed light green regions represent the blocks selected in each chromosome using the indirect approach based on HapMap data. Shadowed dark green boxes represent the location for maximum singlepoint values.

a Representation of the region on chromosome 11.

b Representation of the region on chromosome 14
A

FAM 153

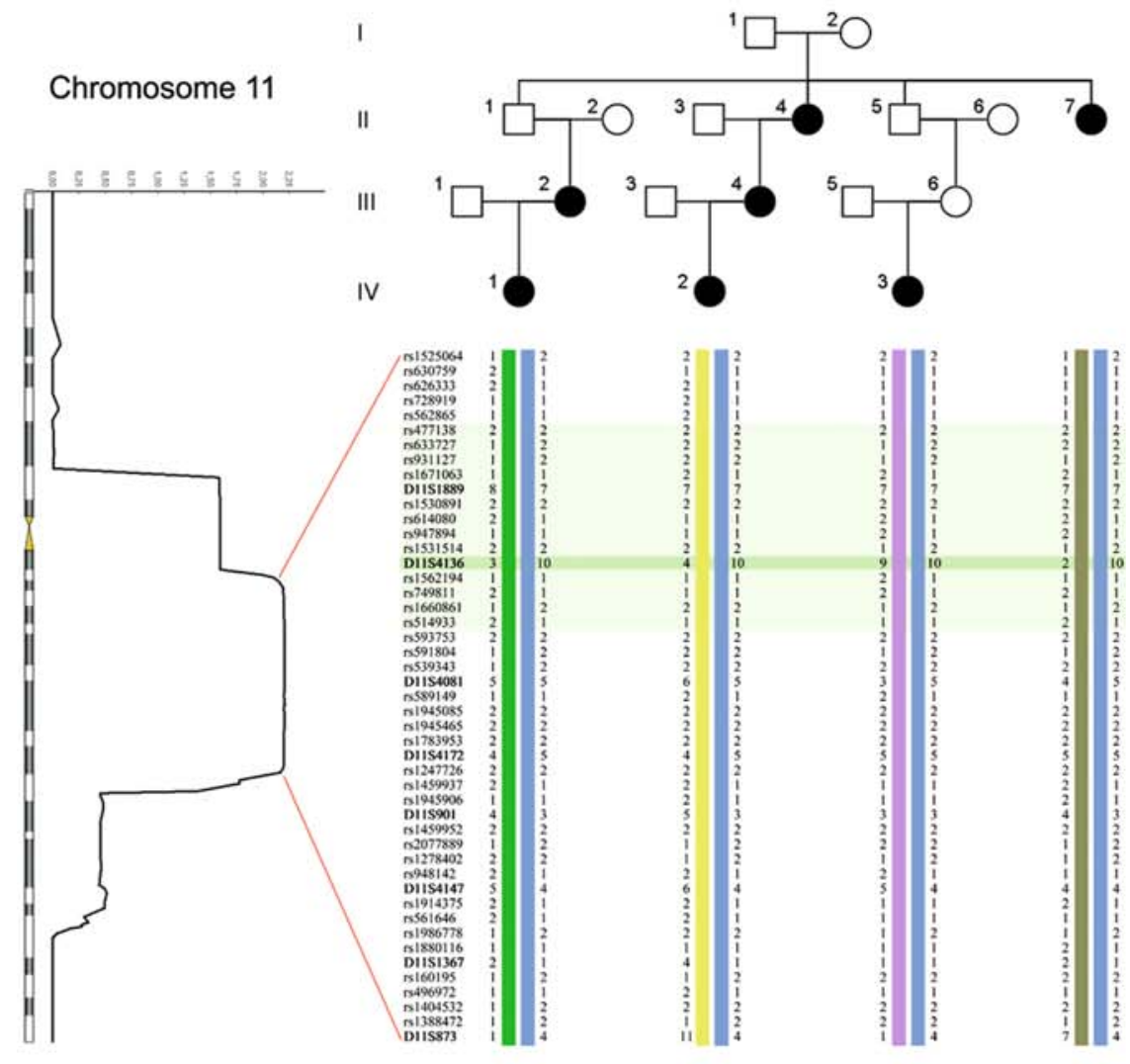

B

FAM 153

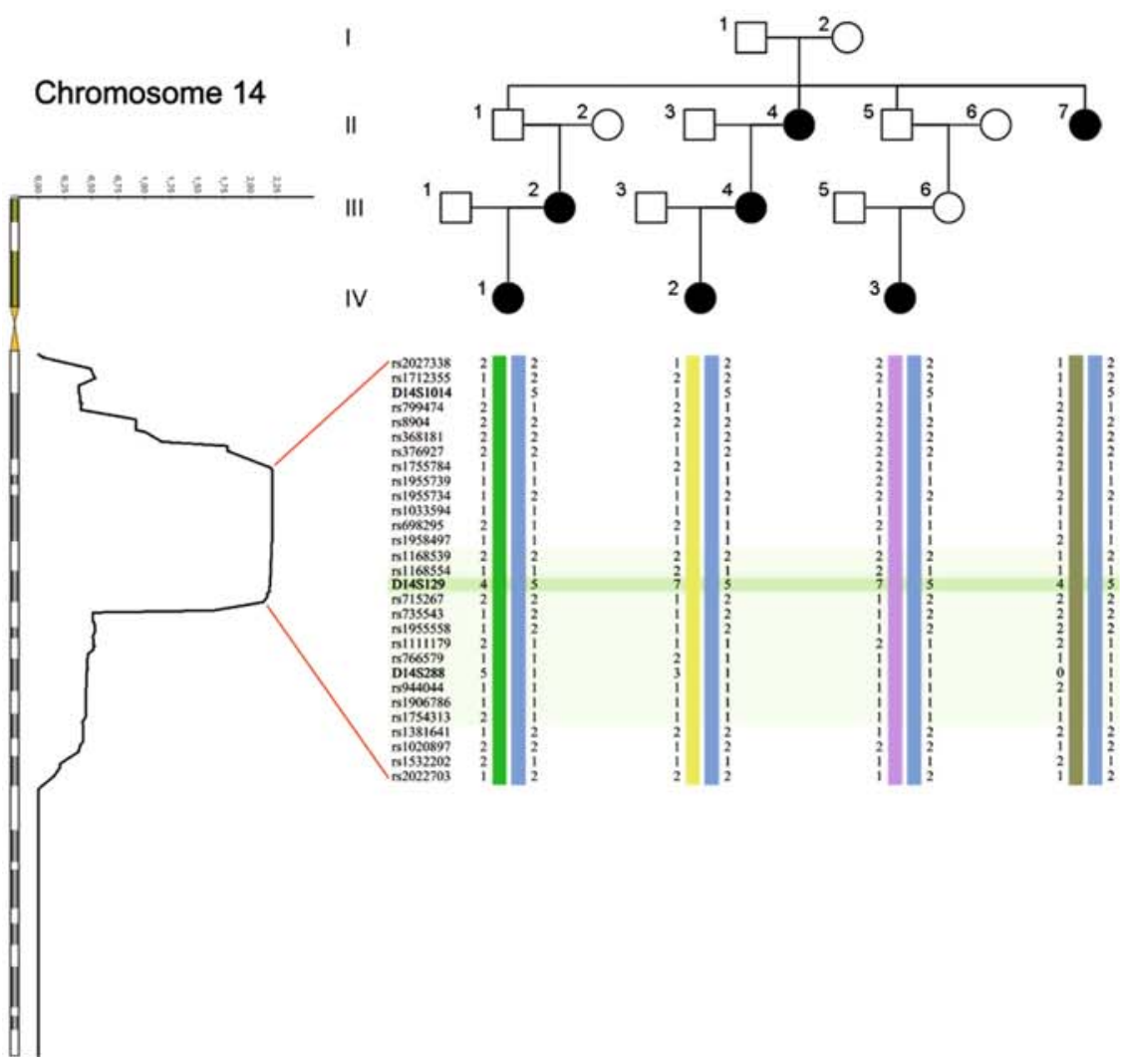



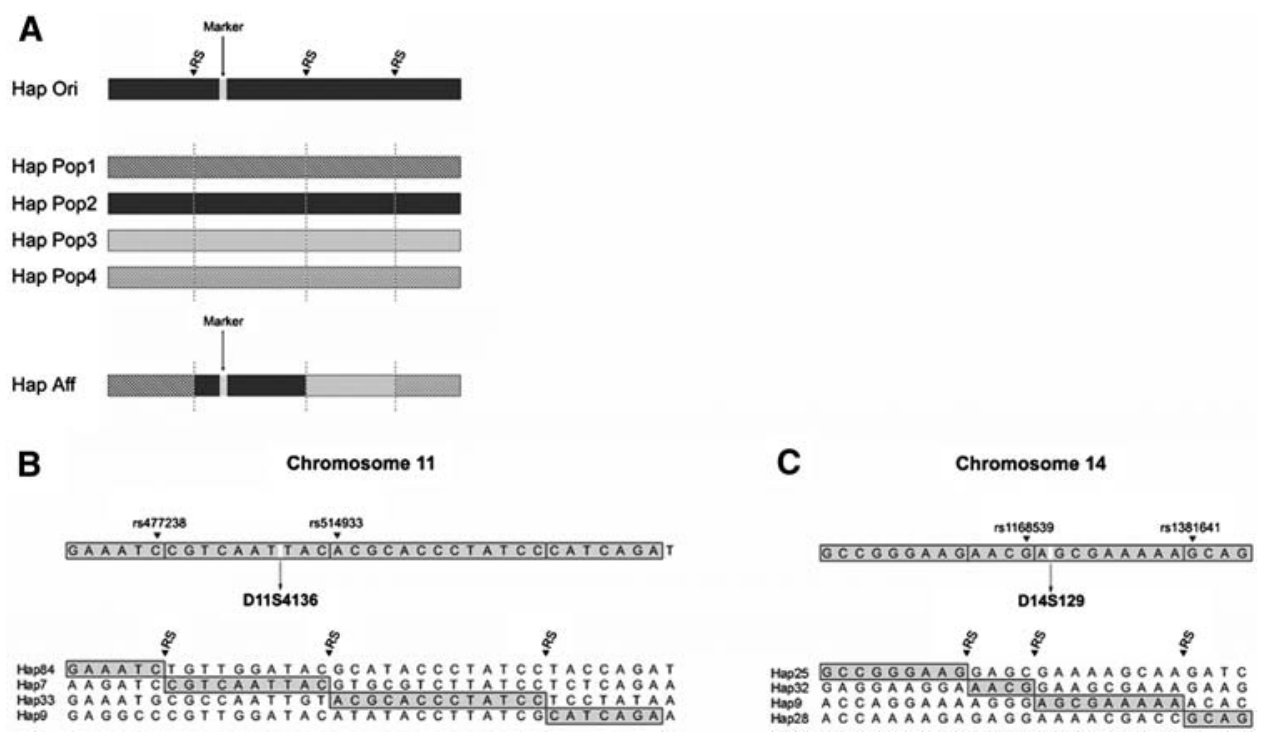

Fig. 2 Representation of the indirect approach using HapMap data to narrow down the candidate regions. a Theoretical representation of the approach. The ancestral haplotype (Hap Ori) showing putative recombination spots $(R S)$ and the putative marker linked to the causal mutation (grey box). Different groups of haplotypes imputed from the Caucasian population (Hap Pop $N$ ) are used to identify the possible recombination spots. The derived haplotype (Hap Aff) shows different blocks of SNPs (estimated based on the putative recombination spots by comparing to HapMap haplotypes) and the marker linked to the causal mutation (grey box). b Diagram used to identify the blocks of

through the generations, since the disease allele appeared in the germ line of a common ancestor (Fig. 2a). It was based on HapMap data from 30 Caucasian trios (http://hapmap. org/cgi-perl/gbrowse/hapmap_B35/) for all the SNP markers in the candidate region that are included in the Illumina Linkage Panel III. We used these haplotype data to select the most probable combination of haplotypes that shared haplotype blocks with our putative risk haplotypes. We then selected the haplotype blocks that contained the STRs that showed the maximum singlepoint LOD scores in our candidate regions in chromosomes 11 and 14 (Fig. 2b, c). The aim of this approach was to obtain the minimal regions from the ancestral chromosomes carried through to the observed chromosomes, assuming the limitations that have been reported to be intrinsic to the estimation of haplotypes using HapMap data for a group of SNPs not in strong LD (limited number of chromosomes, missing genotypes, double heterozygotes and double recombination events).

\section{Gene selection}

Although we reduced the size of the candidate regions, the high number of loci within them forced us to establish two criteria for the selection of genes on which to performing mutational screening: (a) close proximity to the maximum
SNPs within the region in chromosome 11. The affected haplotype is divided into four blocks. There are four different groups of HapMap haplotypes (one representative haplotype is shown for each group) used to obtain three putative recombination spots $(R S)$. The white box represents the location of the maximum singlepoint LOD score (D11S4136). rs477238 and $r s 514933$ mark the limits of the minimal candidate region. c Diagram for the region on chromosome 14. The white box represents the location of the maximum singlepoint LOD score (D14S129). $r s 1168539$ and $r s 1381641$ mark the limits of the minimal candidate region

singlepoint LOD score, and (b) previous publication of function-, expression- and/or cancer-related information. This information was obtained from the NCBI database (http://www.ncbi.nlm.nih.gov/sites/entrez). The selected genes and their functional descriptions are summarized in Table 1 . We firstly selected NUMAI in chromosome 11 and FOXAl in chromosome 14 as candidate genes. Five further genes (CCND1, FADD, FGF3, RNF121, and RAD9A) and one microRNA (hsa-mir-192, information extracted from http://microrna.sanger.ac.uk/cgi-bin/targets/ v3/search.pl) were selected within the 11q13 region.

\section{Gene study}

For all the selected genes, mutational screening was carried out by direct sequencing on an ABI Prism 3700 sequencer. Reference sequences for all coding and UTR regions of the genes were obtained from the Genome database (www. ensembl.org). Primers were designed with at least $20 \mathrm{bp}$ from flanking intronic sequences using Primer3 (http:// frodo.wi.mit.edu/cgi-bin/primer3/primer3_www.cgi). The sequencing was performed on DNA from individual IV-1 using the forward oligonucleotide, and where necessary (for example, where intron-exon boundaries were not welldefined), the sequencing was repeated using the reverse oligonucleotide. When a rare variant in the sequence was 
Table 1 Description of the sequenced genes in the candidate regions in chromosomes 11 and 14

\begin{tabular}{|c|c|c|c|c|}
\hline Chromosome & Location & Gene & Position & Function \\
\hline \multirow[t]{8}{*}{11} & $11 q 13.1$ & hsa-mir-192 & 64415185 & MicroRNA supposed to regulates cancer-related genes \\
\hline & $11 \mathrm{q} 13.1-\mathrm{q} 13.2$ & $R A D 9 A$ & 66915999 & $\begin{array}{l}\text { Cell cycle checkpoint protein required for cell cycle arrest and DNA } \\
\text { damage repair in response to DNA damage }\end{array}$ \\
\hline & $11 q 13.3$ & $C C N D 1$ & 69165054 & $\begin{array}{l}\text { Member of the cyclin family and mutations, amplification and } \\
\text { overexpression of this gene are observed frequently in a variety of } \\
\text { tumors and may contribute to tumorigenesis }\end{array}$ \\
\hline & $11 \mathrm{q} 13.3$ & D11S4136 & 69324892 & Microsatellite which shows maximum singlepoint value \\
\hline & $11 \mathrm{q} 13.3$ & $F G F 3$ & 69333917 & $\begin{array}{l}\text { Member of the fibroblast growth factor family and the closest gene } \\
\text { to D11S4136 }\end{array}$ \\
\hline & $11 \mathrm{q} 13.3$ & $F A D D$ & 69726917 & $\begin{array}{l}\text { Adaptor molecule that interacts with various cell surface receptors } \\
\text { and mediates cell apoptotic signals }\end{array}$ \\
\hline & $11 \mathrm{q} 13.4$ & $R N F 121$ & 71317731 & $\begin{array}{l}\text { Gene with unknown function which contains a Ring Finger Domain } \\
\text { supposed to be involved in DNA-protein and protein-protein } \\
\text { interactions }\end{array}$ \\
\hline & $11 \mathrm{q} 13.4$ & NUMA1 & 71391559 & Mitotic checkpoint protein previously associated with breast cancer \\
\hline \multirow[t]{2}{*}{14} & $14 q 12-q 13$ & FOXA1 & 37128947 & Transcription factor assumed to be a BRCA1 partner \\
\hline & $14 q 21.1$ & D14S129 & 38142033 & Microsatellite which shows maximum singlepoint value \\
\hline
\end{tabular}

STR markers from the fine mapping strategy that showed significant singlepoint LOD scores are shown in bold

found, PCR amplification was repeated and checked for the four members of the family.

All variants found in the selected genes had been previously described in the databases (Ensembl, NCBI), with the exception of an indel of $6 \mathrm{bp}$ within intron 12-13 of NUMA1 (rs11278712), which had no allelic frequencies described in any population.

In order to estimate the allelic frequency of this variant and to assess its effect, we genotyped it in 465 controls and 125 BRCAX probands using DHPLC. To confirm the genotypes of the samples, we sequenced four subjects from each DHPLC profile.

\section{Additional microsatellites in 11q13}

We divided the candidate region in chromosome 11 using the indirect approach based on HapMap data. We selected the block defined between rs477138 and rs514933 based on the location of D11S4136, which showed the maximum singlepoint value (Fig. 3). In order to confirm this region as the most probable candidate region, we used a set of three STRs with high heterozygosity. We selected D11S987 (at $67.64 \mathrm{Mb}$, het =0.82), D11S4113 (at $68.52 \mathrm{Mb}$, het $=0.81$ ) and D11S4139 (at $70.18 \mathrm{Mb}$, het $=0.87$ ) (Fig. 3). The allelic frequencies of these STRs in our population were calculated by genotyping 91 healthy controls (a total of 178 chromosomes for each marker).

We performed parametric analysis based on the markers previously studied plus these three additional STRs using Merlin software.

\section{Results}

\section{Significance testing}

In our previous linkage study [12], we found that a single family showed suggestive linkage signals in two different chromosomes, 11 and 14. We estimated the empirical probability of obtaining two peaks with multipoint HLOD $>2.20$ for a single family by chance to be 0.024 . We estimated the probability that two independent loci could segregate in this family, with ten meioses, to be 0.000002 .

Because finding two regions with high multipoint HLOD segregating in a single family is not expected by chance, we tested for a possible translocation between chromosomes 11 and 14 using both conventional karyotyping of G-bands and FISH analysis. Both analyses suggested that there was not a translocation between the two chromosomes (Fig. 4).

\section{Candidate regions}

We used a panel of 11 STRs in chromosome 11 and 6 STRs in chromosome 14 in order to confirm and narrow down the candidate regions. We also calculated the singlepoint HLOD score for each of these STRs, and obtained maximum singlepoint HLOD scores of 1.32 for D11S4136 in chromosome 11 and 1.33 for D14S129 in chromosome 14 (Fig. 1).

We used an indirect approach with HapMap data to narrow down the candidate regions (see section "Materials and methods" and Fig. 2a). We expected to observe a large 
Fig. 3 Results from the fine mapping of the $11 \mathrm{q} 13$ region with STRs. The columns show the markers, physical distance $(\mathrm{Mb})$, multipoint parametric LOD scores, singlepoint parametric LOD scores and genes sequenced. Markers that represent the limits of the region, obtained by the HapMap-based strategy, are shaded in dark grey. The original fine mapping markers are underlined. Additional markers used to confirm the reduced candidate region (shaded) are shown in italics and identified with an arrow. Markers with significant singlepoint LOD scores are shown in bold

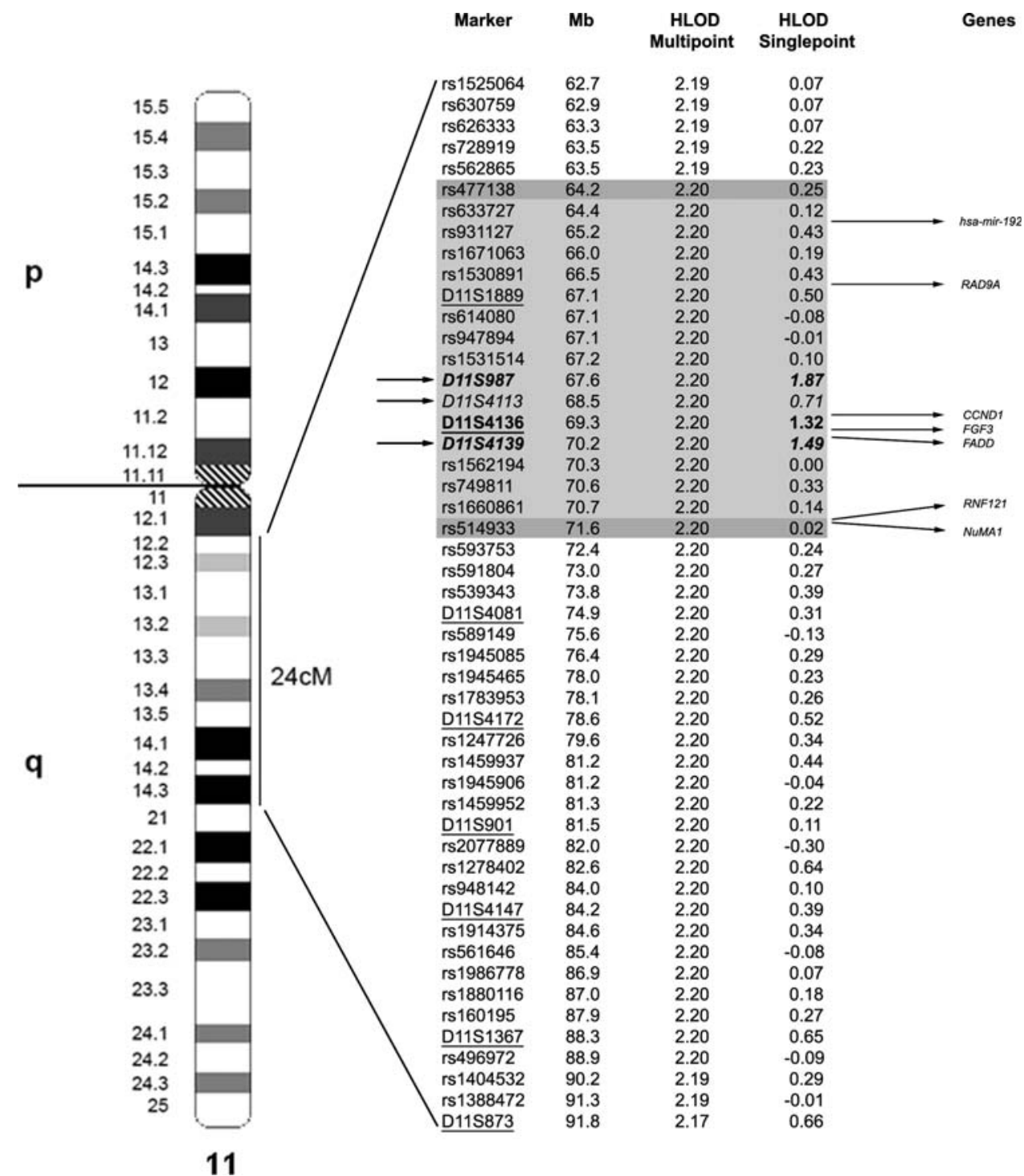

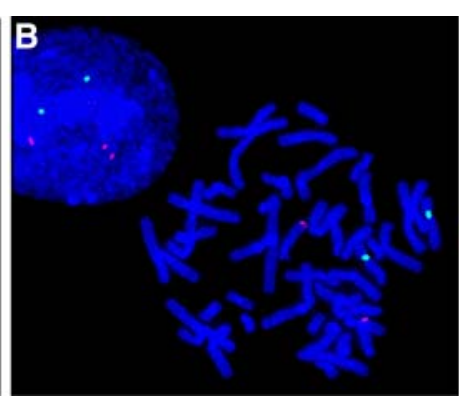

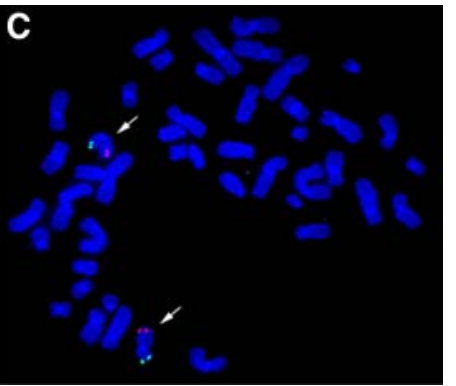

Fig. 4 Cytogenetic analyses used to rule out any cryptic translocation between chromosomes 11 and 14. a Conventional GTG-banding of chromosomes 11 and 14 shows a normal structure for both chromosomes. b FISH analysis using probes specific for the $14 \mathrm{q}$

number of different haplotypes formed by SNPs in linkage disequilibrium blocks. The study of $30 \mathrm{CEPH}$ trios showed a total of 167 different haplotypes for the region in chromosome 11 (formed by 38 SNPs), and 256 (formed by 26 subtelomere (red) and 11 centromere (green). c FISH analysis using probes specific for the $11 \mathrm{p}$ subtelomere (green) and 11q subtelomere (red)

SNPs) for the region in chromosome 14, and none of them were identical to the corresponding segregating risk haplotypes. We identified 4 LD blocks of SNPs in chromosomes 11 and 14, and for each chromosome, we 
selected the block in which the marker with the maximum singlepoint LOD scores was located (Fig. 2b, c).

With this approach, we were able to narrow down the candidate region in chromosome 11 from $28 \mathrm{Mb}$ (424 genes) to $7 \mathrm{Mb}$ (202 genes, from rs477138 to rs514933). On the other hand, the candidate region in chromosome 14 which originally spanned $14.5 \mathrm{Mb}$ (90 genes) was narrowed to $8.3 \mathrm{Mb}$ (38 genes, from rs1168539 to rs1381641) (see Fig. 1). This reduction in the size of the candidate regions allows for more directed and affordable mutational screening.

\section{Candidate gene sequencing}

According to the criteria described in "Materials and Methods", we first selected NUMAI and FOXAl as candidate genes for sequencing in the index case of FAM153. NUMA1 is located on 11q13.4 (see Table 1) and FOXAl is located on 14q12-q13 (see Table 1). The sequencing of the coding region of NUMAI and FOXAl showed no new variation (supplementary table 1 ) from the reference sequence in the databases (Ensembl, NCBI). The only exception to this was an indel of 6 bp identified within intron 12-13 of NUMA1 (rs11278712), for which no allelic frequencies were described in the databases.

To assess whether the derived allele was a polymorphism or a deleterious mutation, we examined its segregation in FAM153, and we confirmed its co segregation with breast cancer disease status (Table 2). Then, we genotyped this indel 465 control samples, and found 42 samples carrying the insertion allele, given an estimated minor allele frequency of 0.045 . This result indicates that the derived allele is not a high-penetrance mutation. We extended this study to the probands from 125 BRCAX families and we found that the minor allele frequency was 0.028 , very similar that among controls $(P=0.24)$.

Further genetic screening of 11q13

After the sequencing of NUMAI and FOXA1, we focused our efforts on the mutational screening of the $11 \mathrm{q} 13$ region. The main reason for this was the observed segregation of the indel allele with breast cancer in the family, suggesting the existence of a causal gene close to NUMA1. In addition, we found familial segregation of a set of rare alleles for three other SNPs within NUMA1: rs642573, rs1057992 and rs1541304 (see Table 2).

Following the selection criteria established in the material and methods section, we selected another five genes and one miRNA close to D11S4136, all within the $7 \mathrm{Mb}$ region (Table 1; Fig. 3). We sequenced the coding and UTR regions of FGF3, CCND1, FADD and hsa-mir-192, but no new variation was found (supplementary table 1).

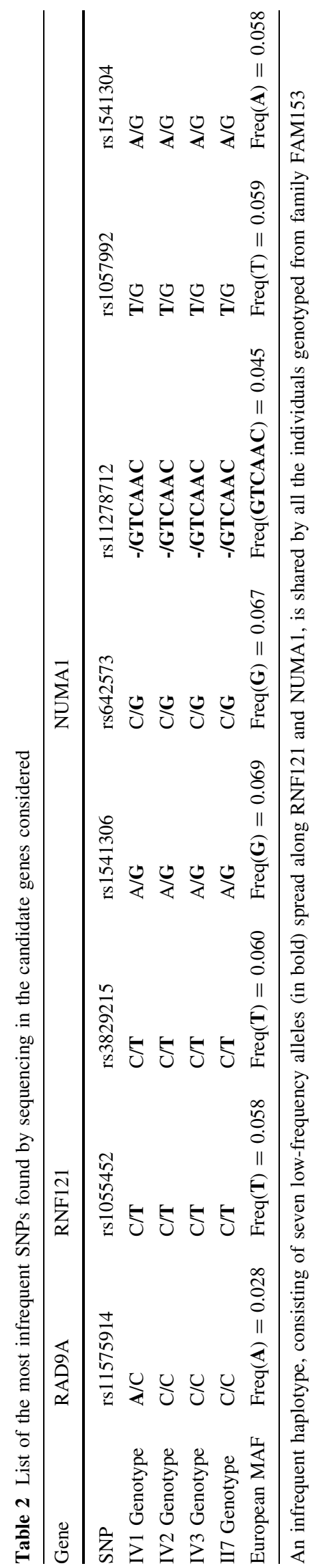


We also selected $R N F 121$ to be sequenced because its functional domains predict a protein-DNA, protein-protein interacting molecule and it is located on the same LD block that NUMAI (data not shown). Although we did not find any new variation in the coding region of $R N F 121$, we observed that this family shared a group of very infrequent alleles for seven variants found in NUMA1 and RNF121 (see Table 2), three of them in intronic regions of NUMA1 (rs642573, rs1057992 and rs11278712), one of them in the $3^{\prime} \mathrm{UTR}$ regions of NUMA1 (rs1541304) and other three in the $3^{\prime} \mathrm{UTR}$ of RNF121 (rs1055452, rs3829215 and rs1541306) (see Table 2).

We genotyped these seven rare variants in five control samples that were heterozygous for the indel allele. We found that this rare haplotype likely appeared in four of these five controls. Thus, we estimated the minimum frequency of this haplotype to be greater than $3.6 \%$ in the general population. We therefore ruled out that this haplotype is deleterious for familial breast cancer.

As a final step in the mutational screening within 11q13, we sequenced $R A D 9 A$, but we did not find any new variation. However, we found that the index case of the family carried the minor A allele for rs11575914, which is described as having a frequency of 0.028 in the general population. We genotyped this SNP in the four individuals of the family, and found that the A allele did not segregate with breast cancer (see Table 2).

Confirmation of the $7 \mathrm{Mb}$ candidate region (from rs477138 to rs514933)

In order to confirm the segregation of this region in family FAM153, we genotyped three STRs close to D11S4136 (Fig. 3). We observed that the allelic segregation of D11S987, D11S4113 and D11S4139 was consistent with the haplotype being shared by all genotyped members in the family. We then performed a parametric analysis using the data from our previous linkage study [12], and found that D11S987 and D11S4139 had even higher singlepoint values than D11S4136, which confirms that this region is the most likely candidate to carry the disease locus (Fig. 3).

\section{Discussion}

In the present study we have extended our previous work and described in detail two candidate regions to carry a breast cancer susceptibility gene. One region is located on chromosome 11 and spans $7 \mathrm{Mb}$. The other region is located on chromosome 14 and spans 8.5 Mb. Moreover, several genes within the $11 \mathrm{q} 13$ and $14 \mathrm{q} 12-\mathrm{q} 21$ regions have been screened but no deleterious mutations have been found. It should be noted that we cannot rule out the possibility of mutations affecting the regulation of these genes (e.g., in promoter region) or large rearrangements, because sequencing was confined to the coding regions only.

Family FAM153 showed suggestive linkage to two peaks in two different chromosomes. We performed a simulation study in our set of families to estimate empirically the probability of finding a family with suggestive linkage in two different chromosomes, by chance along. This was statistically significant $(P=0.024)$, thus we assumed that two different high-risk loci could cosegregate in this family. In addition, we estimated the probability the two independent loci segregate together through the ten meioses observed in this family, and it was very low $(1 / 524,288)$. However, some cases of familial segregation of mutations in both $B R C A 1$ and $B R C A 2$ have been reported $[14,15]$, highlighting that the transmission of mutations in two different and independent high-penetrance genes through multiple generations in a single family is possible, although very infrequent. Finally, we assessed whether a germinal translocation between both chromosome 11 and chromosome 14 exists, which could explain the familial segregation of these two regions, and the results confirmed the absence of any such translocation (Fig. 4).

Each of these two regions could therefore contain a high-risk breast cancer gene. Since we have not found any mutations in the genes considered, we cannot rule out that only one or neither of them contains a causal gene. We similarly cannot exclude the possibility that two moderate penetrance genes at each locus may interact, either with each other, or each with other low penetrance variants.

Due to the large size of the candidate regions $(28 \mathrm{Mb}$ in chromosome 11 and $14.5 \mathrm{Mb}$ in chromosome 14), we applied both a fine mapping strategy based on STRs and an indirect approach based on HapMap data to narrow down these regions. The markers with the highest associated singlepoint LOD scores were the microsatellites, because they present greater heterozygosity than SNP markers and are therefore more informative. Using this strategy, we established that the maximum singlepoint LOD score was for D11S4136 in chromosome 11 and for D14S129 in chromosome 14 (Fig. 1). In addition, we applied an indirect approach to narrow down the affected shared region in both chromosomes using HapMap data from the Caucasian population (see section "Materials and Methods").

We first selected NUMA1, in chromosome 11, and FOXA1, in chromosome 14, as candidate genes for sequencing. NUMAl has been previously described as a putative tumor suppressor gene associated with breast cancer and FOXAl belongs to an extensive transcription factor family spread across the genome (Table 1). The sequencing did not reveal any deleterious germline mutations in the coding regions of these genes, with the exception of an insertion of six base pairs (rs11278712) in 
NUMA1 that was subsequently found to segregate in the family. Although we have ruled out its possible role as a high penetrance mutation, the presence of this marker segregating with breast cancer suggests that this chromosomal region may contain a putative causal gene. We therefore focused our efforts on further mutational screening of region $11 \mathrm{q} 13$.

Region 11q13 is considered to be important in cancer development, and specifically in breast cancer progression, and amplifications as well as deletions have been described [16-18]. Thus, we selected five other genes and one microRNA for mutational screening, based on functional as well as expression studies and distance to the STR with maximum singlepoint HLOD score (Table 1). Although we found different variants in both coding and non-coding regions, none were deleterious mutations (supplementary table 1).

Since the mutational screening did not reveal any deleterious mutations, we sought to confirm that region rs477138-rs514933 in chromosome 11 was the most likely candidate region. Therefore, we genotyped three additional STRs with high heterozygosty, close to D11S4136, and found that two of them gave high singlepoint LOD scores, which suggests that the region is a strong candidate to carry the disease locus (Fig. 2). The 11q13 region has a high density of genes and our final candidate region of $7 \mathrm{Mb}$ contains around 200 genes. The unknown function of most of the genes in the region may have biased the selection we made, since it was based on know functional features. Further mutational screening within this region is therefore required.

In conclusion, our results suggest that the $11 \mathrm{q} 13$ region might carry a breast cancer susceptibility gene. We have narrowed it down from 28 to $7 \mathrm{Mb}$ using both a fine-mapping study and an indirect approach based on HapMap data. We have performed mutational screening in some candidate genes, but have not found any deleterious mutations. A larger study involving additional families linked to this region is required to further narrow down the region and facilitate a more extensive mutational screening to find a causal gene.

Acknowledgments We thank David Rodriguez from the Genomics Unit at the Spanish National Cancer Research Centre, for his technical support in sequencing. We also thank Lorenzo Melchor and Ricardo Ramires from the Human Genetics Group for their scientific support and advice. This study has been funded by project FIS-042240 from the Fondo de Investigaciones Sanitarias, and by the Asociación Española Contra el Cáncer. JMRR Grant sponsor: Ministerio de Educacion y Ciencia, FPU AP-2005-1720.

\section{References}

1. Nathanson KL, Wooster R, Weber BL (2001) Breast cancer genetics: what we know and what we need. Nat Med 7:552-556. doi:10.1038/87876
2. Casey G (1997) The BRCA1 and BRCA2 breast cancer genes. Curr Opin Oncol 9:88-93

3. Easton D (1997) Breast cancer genes-what are the real risks? Nat Genet 16:210-211. doi:10.1038/ng0797-210

4. Diez O, Osorio A, Duran M et al (2003) Analysis of BRCA1 and BRCA2 genes in Spanish breast/ovarian cancer patients: a high proportion of mutations unique to Spain and evidence of founder effects. Hum Mutat 22:301-312. doi:10.1002/humu.10260

5. Walsh T, Casadei S, Coats KH et al (2006) Spectrum of mutations in BRCA1, BRCA2, CHEK2, and TP53 in families at high risk of breast cancer. JAMA 295:1379-1388. doi:10.1001/jama. 295.12.1379

6. Smith P, McGuffog L, Easton DF et al (2006) A genome wide linkage search for breast cancer susceptibility genes. Genes Chromosomes Cancer 45:646-655. doi:10.1002/gcc.20354

7. Huusko P, Juo SH, Gillanders E et al (2004) Genome-wide scanning for linkage in Finnish breast cancer families. Eur J Hum Genet 12:98-104. doi:10.1038/sj.ejhg.5201091

8. Thompson D, Szabo CI, Mangion J et al (2002) Evaluation of linkage of breast cancer to the putative BRCA3 locus on chromosome $13 \mathrm{q} 21$ in 128 multiple case families from the breast cancer linkage consortium. Proc Natl Acad Sci USA 99:827-831. doi:10.1073/pnas.012584499

9. Bergman A, Karlsson P, Berggren J et al (2007) Genome-wide linkage scan for breast cancer susceptibility loci in Swedish hereditary non-BRCA1/2 families: suggestive linkage to 10q23.32-q25.3. Genes Chromosomes Cancer 46:302-309. doi: $10.1002 /$ gcc. 20405

10. Pharoah PD, Antoniou A, Bobrow M et al (2002) Polygenic susceptibility to breast cancer and implications for prevention. Nat Genet 31:33-36. doi:10.1038/ng853

11. Honrado E, Osorio A, Milne RL et al (2007) Immunohistochemical classification of non-BRCA1/2 tumors identifies different groups that demonstrate the heterogeneity of BRCAX families. Mod Pathol 20:1298-1306. doi:10.1038/modpathol. 3800969

12. Gonzalez-Neira A, Rosa-Rosa JM, Osorio A et al (2007) Genomewide high-density SNP linkage analysis of non-BRCA1/2 breast cancer families identifies various candidate regions and has greater power than microsatellite studies. BMC Genomics 8:299. doi:10.1186/1471-2164-8-299

13. Abecasis GR, Cherny SS, Cookson WO et al (2002) Merlinrapid analysis of dense genetic maps using sparse gene flow trees. Nat Genet 30:97-101. doi:10.1038/ng786

14. Caldes T, de la Hoya M, Tosar A et al (2002) A breast cancer family from Spain with germline mutations in both the BRCA1 and BRCA2 genes. J Med Genet 39:e44. doi:10.1136/jmg. 39.8.e44

15. Liede A, Rehal P, Vesprini D et al (1998) A breast cancer patient of Scottish descent with germ-line mutations in BRCA1 and BRCA2. Am J Hum Genet 62:1543-1544. doi:10.1086/301889

16. Buness A, Kuner R, Ruschhaupt M et al (2007) Identification of aberrant chromosomal regions from gene expression microarray studies applied to human breast cancer. Bioinformatics 23:22732280. doi:10.1093/bioinformatics/btm340

17. Loo LW, Grove DI, Williams EM et al (2004) Array comparative genomic hybridization analysis of genomic alterations in breast cancer subtypes. Cancer Res 64:8541-8549. doi:10.1158/00085472.CAN-04-1992

18. Paterson AL, Pole JC, Blood KA et al (2007) Co-amplification of $8 \mathrm{p} 12$ and $11 \mathrm{q} 13$ in breast cancers is not the result of a single genomic event. Genes Chromosomes Cancer 46:427-439. doi: $10.1002 /$ gcc. 20424 